\title{
System Analysis via Integral Quadratic Constraints
}

\author{
Alexandre Megretski, Member, IEEE, and Anders Rantzer, Member, IEEE
}

\begin{abstract}
This paper introduces a unified approach to robustness analysis with respect to nonlinearities, time variations, and uncertain parameters. From an original idea by Yakubovich, the approach has been developed under a combination of influences from the Western and Russian traditions of control theory.

It is shown how a complex system can be described, using integral quadratic constraints (IQC's) for its elementary components. A stability theorem for systems described by IQC's is presented that covers classical passivity/dissipativity arguments but simplifies the use of multipliers and the treatment of causality. A systematic computational approach is described, and relations to other methods of stability analysis are discussed. Last, but not least, the paper contains a summarizing list of IQC's for important types of system components.
\end{abstract}

Index Terms - Nonlinearity, robustness, stability analysis.

\section{INTRODUCTION}

$\mathbf{I}$ T IS common engineering practice to work with the simplest possible models in design of control systems. In particular, one often uses linear time-invariant plant models, for which there exist a well-established theory and commercially available computer tools that help in the design. Experiments, often preceded by simulations with more accurate models, are used to verify that the design also works well in practice. There is also a strong need for more formal ways to analyze the systems. Such analysis can help to identify critical experimental circumstances or parameter combinations and estimate the power of the models.

In the 1960-70's, a large body of results was developed in this direction, often referred to as "absolute stability theory." The basic idea was to partition the system into a feedback interconnection of two positive operators; see [1]-[7] and the references therein. To improve the flexibility of the approach, so-called multipliers were used to select proper variables for the partitioning. The absolute stability theory is now considered a fundamental component of the theory for nonlinear systems. However, the applicability of many of the results has been limited by computational problems and by restrictive causality conditions used in the multiplier theory.

For computation of multipliers, substantial progress has been made in the last decade, the most evident example being algorithms for computation of structured singular values $(\mu$

Manuscript received October 5, 1995; revised September 6, 1996. Recommended by Associate Editor, A. Tesi. This work was been supported by the National Science Foundation under Grant ECS9410531 and the Swedish Research Council for Engineering Sciences under Grant 94-716. The paper was written while A. Megretski was on the faculty of Iowa State University.

A. Megretski is with the Department of Electrical Engineering, MIT, Cambridge, MA 02139 USA (e-mail: ameg@ mit.edu).

A. Rantzer is with the Department of Automatic Control, Lund Institute of Technology, S-221 00 Lund, Sweden.

Publisher Item Identifier S 0018-9286(97)04274-8. analysis) [8]. As a result, robustness analysis with respect to uncertain parameters and unmodeled dynamics can be performed with great accuracy. An even more fundamental breakthrough in this direction is the development of polynomial time algorithms for convex optimization with constraints defined by linear matrix inequalities [9], [10]. Such problems appear not only in $\mu$-analysis but also in almost any analysis setup based on concepts of passivity-type.

The purpose of this paper is to address the second obstacle to efficient analysis by proving that multipliers can be introduced in a less restrictive manner, without causality constraints. Not only does this make the theory more accessible by simplification of proofs, but it also enhances the development of computer tools that support the transformation of model structure assumptions into numerically tractable optimization problems.

The concept integral quadratic constraint (IQC) is used for several purposes:

- to exploit structural information about perturbations;

- to characterize properties of external signals;

- to analyze combinations of several perturbations and external signals.

Implicitly, IQC's have always been used in stability theory. For example, positivity of an operator $F$ can be expressed by the IQC

$$
\int_{-\infty}^{\infty} \widehat{(F v)}(j \omega)^{*} \widehat{v}(j \omega) d \omega \geq 0, \quad \forall v
$$

In the 1960's, most of the stability theory was devoted to scalar feedback systems. This led to conveniently visualizable stability criteria based on the Nyquist diagram, which was particularly important in times when computers were less accessible.

In the 1970's, IQC's were explicitly used by Yakubovich to treat the stability problem for systems with advanced nonlinearities, including amplitude and frequency modulation systems. Some new IQC's were introduced, and the so-called $S$-procedure was applied to the case of multiple constraints [54]. Willems also gave an energy-related interpretation of the stability results, in terms of dissipativity, storage functions, and supply rates [4]. Later on, Safonov interpreted the stability results geometrically, in terms of separation of the graphs of the two operators in the feedback loop.

An important step in further development was the introduction of analysis methods which essentially rely on the use of computers. One example is the theory for quadratic stabilization [11], [12], and another is the multiloop generalization of the circle criterion based on D-scaling [13], [8]. Both search for a Lyapunov function, and the search for D-scales can be 
interpreted as optimization of parameters in an IQC. Another direction was the introduction of $H^{\infty}$ optimization for the synthesis of robust controllers [14], [15]. Again, the results can be viewed in terms of IQC's, since optimal design with respect to an IQC leads to $H^{\infty}$ optimization.

During the last decade, a variety of methods has been developed within the area of robust control. As was pointed out in [16], many of them can be reformulated to fall within the framework of IQC's. This will be further demonstrated in the current paper, which presents a minimal framework for the stability analysis of feedback interconnections described in terms of IQC's. In Section II, definitions and a main theorem are given in detail. They are illustrated in Section III by an extensive example, analyzing a system with saturation and an uncertain delay. Then, follow sections with discussions and comparisons to well-known results. Finally, we give a summarizing list of IQC's for important types of system components.

\section{A. Notation}

Let $\mathbf{R} \mathbf{L}_{\infty}$ be the set of proper (bounded at infinity) rational functions with real coefficients. The subset consisting of functions without poles in the closed right-half plane is denoted $\mathbf{R H}_{\infty}$. The set of $m \times n$ matrices with elements in $\mathbf{R} \mathbf{L}_{\infty}$ $\left(\mathbf{R H}_{\infty}\right)$ will be denoted $\mathbf{R} \mathbf{L}_{\infty}^{m \times n}\left(\mathbf{R H}_{\infty}^{m \times n}\right)$.

$\mathbf{L}_{2}^{l}[0, \infty)$ can be thought of as the space of $\mathbf{R}^{l}$-valued functions $f:[0, \infty) \rightarrow \mathbf{R}^{l}$ of finite energy

$$
\|f\|^{2}=\int_{0}^{\infty}|f(t)|^{2} d t
$$

This is a subset of the space $\mathbf{L}_{2 \mathrm{e}}^{l}[0, \infty)$, whose members only need to be square integrable on finite intervals. The Fourier transform of $f \in \mathbf{L}_{2}^{l}[0, \infty)$ is denoted by

$$
\hat{f}(j \omega)=\int_{0}^{\infty} e^{-j \omega t} f(t) d t
$$

By an operator we mean a function $F: \mathbf{L}_{2 \mathrm{e}}^{a}[0, \infty) \rightarrow$ $\mathbf{L}_{2 \mathrm{e}}^{b}[0, \infty)$ from one $\mathbf{L}_{2 \mathrm{e}}[0, \infty)$ space to another. The gain of an operator $F: \mathbf{L}_{2 \mathrm{e}}^{a}[0, \infty) \rightarrow \mathbf{L}_{2 \mathrm{e}}^{b}[0, \infty)$ is given by

$$
\|F\|=\sup \left\{\|F(f)\| /\|f\|: \quad f \in \mathbf{L}_{2}^{a}[0, \infty), f \neq 0\right\} .
$$

An important example of an operator is given by the past projection (truncation) $P_{T}$, which leaves a function unchanged on the interval $[0, T]$ and gives the value zero on $(T, \infty]$. Causality of an operator $F$ means that $P_{T} F=P_{T} F P_{T}$ for any $T>0$.

\section{B. What Is an IQC?}

IQC's provide a way of representing relationships between processes evolving in a complex dynamical system, in a form that is convenient for analysis.

Depending on the particular application, various versions of IQC's are available. Two signals $w \in \mathbf{L}_{2}^{m}[0, \infty)$ and $v \in \mathbf{L}_{2}^{l}[0, \infty)$ are said to satisfy the IQC defined by $\Pi$, if

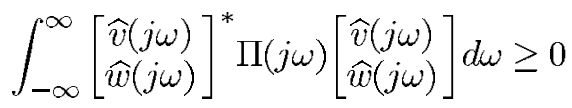

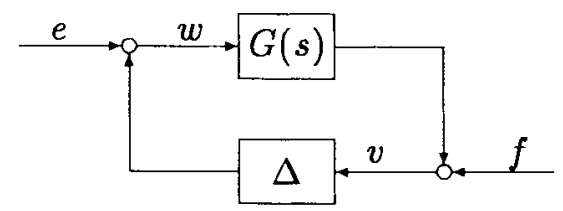

Fig. 1. Basic feedback configuration.

(absolute integrability is assumed). Here the Fourier transforms $\widehat{w}(j \omega)$ and $\widehat{v}(j \omega)$ represent the harmonic spectrum of the signals $u$ and $v$ at the frequency $\omega$, and (1) describes the energy distribution in the spectrum of $(v, w)$. In principle, $\Pi: j \mathbf{R} \rightarrow \mathbf{C}^{(l+m) \times(l+m)}$ can be any measurable Hermitianvalued function. In most situations, however, it is sufficient to use rational functions that are bounded on the imaginary axis.

A time-domain form of (1) is

$$
\int_{0}^{\infty} \sigma\left(x_{\pi}(t), w(t), v(t)\right) d t \geq 0
$$

where $\sigma$ is a quadratic form, and $x_{\pi}$ is defined by

$$
\dot{x}_{\pi}(t)=A_{\pi} x_{\pi}(t)+B_{w} w(t)+B_{v} v(t), \quad x_{\pi}(0)=0
$$

where $A_{\pi}$ is a Hurwitz matrix. Intuitively, this state-space form IQC is a combination of a linear filter (3) and a "correlator" (2). For any bounded rational weighting function $\Pi$, (1) can be expressed in the form (2), (3) by first factorizing $\Pi$ as $\Pi(j \omega)=$ $\Psi(j \omega)^{*} M \Psi(j \omega)$ with $\Psi(j \omega)=C_{\psi}\left(j \omega I-A_{\pi}\right)^{-1}\left[\begin{array}{ll}B_{w} & \left.B_{v}\right]+\end{array}\right.$ $D_{\psi}$, then defining $\sigma$ from $C_{\psi}, D_{\psi}$, and $M$.

In system analysis, IQC's are useful to describe relations between signals in a system component. For example, to describe the saturation $w=\operatorname{sat}(\mathrm{v})$, one can use the IQC defined by (1) with $\Pi=\operatorname{diag}\{1,-1\}$, which holds for any square summable signals $w, v$ related by $w=\operatorname{sat}(\mathrm{v})$. In general, a bounded operator $\Delta: \mathbf{L}_{2 \mathrm{e}}^{l}[0, \infty) \rightarrow \mathbf{L}_{2 \mathrm{e}}^{m}[0, \infty)$ is said to satisfy the IQC defined by $\Pi$ if (1) holds for all $w=\Delta(v)$, where $v \in \mathbf{L}_{2}^{l}[0, \infty)$.

There is, however, an evident problem in using IQC's in stability analysis. This is because both (1) and (2), (3) make sense only if the signals $w, v$ are square summable. If it is not known a priori that the system is stable, then the signals might not be square summable. ${ }^{1}$ This will be resolved as follows. First, the system is considered as depending on a parameter $\tau \in[0,1]$, such that stability is obvious for $\tau=0$, while $\tau=1$ gives the system to be studied. Then, the IQC's are used to show that as $\tau$ increases from zero to one, there can be no transition from stability to instability.

\section{A Basic Stability Theorem}

The following feedback configuration, illustrated in Fig. 1, is the basic object of study in this paper:

$$
\left\{\begin{array}{l}
v=G w+f \\
w=\Delta(v)+e .
\end{array}\right.
$$

\footnotetext{
${ }^{1}$ One could suggest using integrals "from 0 to $T$ " in (2) instead of the integrals "from 0 to $\infty$," as is often done in the literature. It can be shown, however, that for many important components (such as a saturation), some useful IQC's hold in the form (2), but their counterparts "from 0 to $T$ " are not valid (see Section IV).
} 
Here $f \in \mathbf{L}_{2 \mathrm{e}}^{l}[0, \infty), e \in \mathbf{L}_{2 \mathrm{e}}^{m}[0, \infty)$ represent the "interconnection noise," and $G$ and $\Delta$ are the two causal operators on $\mathbf{L}_{2 \mathrm{e}}^{m}[0, \infty)$ and $\mathbf{L}_{2 \mathrm{e}}^{l}[0, \infty)$, respectively. It is assumed that $G$ is a linear time-invariant operator with the transfer function $G(s)$ in $\mathbf{R H}_{\infty}^{l \times m}$, and $\Delta$ has bounded gain.

In applications, $\Delta$ will be used to describe the "troublemaking" (nonlinear, time-varying, or uncertain) components of a system. The notation $G$ will either denote a linear operator or a rational transfer matrix, depending on the context. The following definitions will be convenient.

Definition: We say that the feedback interconnection of $G$ and $\Delta$ is well-posed if the map $(v, w) \mapsto(e, f)$ defined by (4) has a causal inverse on $\mathbf{L}_{2 \mathrm{e}}^{m+l}[0, \infty)$. The interconnection is stable if, in addition, the inverse is bounded, i.e., if there exists a constant $C>0$ such that

$$
\int_{0}^{T}\left(|v|^{2}+|w|^{2}\right) d t \leq C \int_{0}^{T}\left(|f|^{2}+|e|^{2}\right) d t
$$

for any $T \geq 0$ and for any solution of (4).

When $G$ is linear, as will be the case below, well-posedness means that $I-G \Delta$ is causally invertible. From boundedness of $G$ and $\Delta$, it also follows that the interconnection is stable if and only if $(I-G \Delta)^{-1}$ is a bounded causal operator on $\mathbf{L}_{2}^{l}[0, \infty)$.

In most applications, well-posedness is equivalent to the existence, uniqueness, and continuability of solutions of the underlying differential equations and is relatively easy to verify. Regarding stability, it is often desirable to verify some kind of exponential stability. However, for general classes of ordinary differential equations, exponential stability is equivalent to the input/output stability introduced above (compare [39, sec. 6.3]).

Proposition 1: Consider $\phi$ with $\sup _{x, t}|\phi(x, t)| /|x(t)|<$ $\infty$. Assume that for any $g \in \mathbf{L}_{2}^{n}[0, \infty), x_{0} \in \mathbf{R}^{n}, t_{0} \geq 0$ the system

$$
\dot{x}(t)=\phi(x(t), t)+g(t), \quad t \geq t_{0}
$$

has a solution $x(\cdot)$. Then the following two conditions are equivalent.

1) There exists a constant $c>0$ such that

$$
\int_{0}^{T}|x(t)|^{2} d t \leq c \int_{0}^{T}|g(t)|^{2} d t, \quad \forall T>0
$$

for any solution of (6) with $x(0)=0$.

2) There exist $\epsilon, d>0$ such that

$$
\left|x\left(t_{1}\right)\right|^{2} \leq d e^{\epsilon\left(t_{0}-t_{1}\right)}\left|x\left(t_{0}\right)\right|^{2}+d \int_{t_{0}}^{t_{1}}|g(t)|^{2} d t
$$

for any solution $x$ of (6).

Proof: Parts, if not all, of this result can be found in standard references on nonlinear systems. A complete proof is also included in the technical report [40] that contains an early version of this paper.
We are now ready to state our main theorem.

Theorem 1: Let $G(s) \in \mathbf{R H}_{\infty}^{l \times m}$, and let $\Delta$ be a bounded causal operator. Assume that:

i) for every $\tau \in[0,1]$, the interconnection of $G$ and $\tau \Delta$ is well-posed;

ii) for every $\tau \in[0,1]$, the IQC defined by $\Pi$ is satisfied by $\tau \Delta$;

iii) there exists $\epsilon>0$ such that

$$
\left[\begin{array}{c}
G(j \omega) \\
I
\end{array}\right]^{*} \Pi(j \omega)\left[\begin{array}{c}
G(j \omega) \\
I
\end{array}\right] \leq-\epsilon I, \quad \forall \omega \in \mathbf{R} .
$$

Then, the feedback interconnection of $G$ and $\Delta$ is stable. Remark 1: The values

$$
\left[\begin{array}{cc}
I & 0 \\
0 & -I
\end{array}\right]
$$

and

$$
\left[\begin{array}{ll}
0 & I \\
I & 0
\end{array}\right]
$$

of $\Pi(i \omega)$ recover versions of the small gain theorem and the passivity theorem.

Remark 2: In many applications (see for example the previous remark), the upper left corner of $\Pi(j \omega)$ is positive semi-definite, and the lower right corner is negative semidefinite so $\tau \Delta$ satisfies the IQC defined by $\Pi$ for $\tau \in[0,1]$ if and only if $\Delta$ does so. This simplifies Assumption ii).

Remark 3: The theorem remains true if the right-hand side of (9) is replaced by $-\epsilon G(j \omega)^{*} G(j \omega)$. This is obtained by replacing $\Pi(i \omega)$ with

$$
\Pi(i \omega)+\left[\begin{array}{cc}
\epsilon /\|\Delta\| & 0 \\
0 & -\epsilon
\end{array}\right] .
$$

The corresponding IQC for $\Delta$ is valid by definition of $\|\Delta\|$.

Remark 4: It is important to note that if $\tau \Delta$ satisfies several IQC's, defined by $\Pi_{1}, \cdots, \Pi_{n}$, then a sufficient condition for stability is the existence of $x_{1}, \cdots, x_{n} \geq 0$ such that (9) holds for $\Pi=x_{1} \Pi_{1}+\cdots+x_{n} \Pi_{n}$. Hence, the more IQC's that can be verified for $\Delta$, the better. Furthermore, the condition is necessary in the following sense. If it fails for all $x_{i} \geq 0$, then (5) fails for some signals $f, v, w$ with $v=G w+f$ and $v, w$ satisfying all the IQC's [17], [18].

Proof of Theorem 1 (Step 1): Show that there exists $c_{0}>0$ such that

$$
\|v\| \leq c_{0}\|v-\tau G \Delta(v)\|, \quad \forall v \in \mathbf{L}_{2}^{l}[0, \infty), \tau \in[0,1] .
$$

Introduce the notation

$$
\sigma_{\Pi}(v, w):=\int_{-\infty}^{\infty}\left[\begin{array}{l}
\widehat{v}(j \omega) \\
\widehat{w}(j \omega)
\end{array}\right]^{*} \Pi(j \omega)\left[\begin{array}{l}
\widehat{v}(j \omega) \\
\widehat{w}(j \omega)
\end{array}\right] d \omega
$$

and let $m_{11}, m_{12}, m_{22}$ be the norms $m_{i j}=\sup _{\omega}\left\|\Pi_{i j}(j \omega)\right\|$ for the matrix blocks of

$$
\Pi(j \omega)=\left[\begin{array}{ll}
\Pi_{11}(j \omega) & \Pi_{12}(j \omega) \\
\Pi_{12}(j \omega)^{*} & \Pi_{22}(j \omega)
\end{array}\right] .
$$


For $\epsilon>0$, let $c(\epsilon)=m_{11}+m_{11}^{2} / \epsilon+m_{12}^{2} / \epsilon$. Then

$$
\begin{aligned}
& \left|\sigma_{\Pi}(v+\delta, w)-\sigma_{\Pi}(v, w)\right| \\
& =\left|\int_{-\infty}^{\infty}\left[\widehat{\delta}^{*} \Pi_{11} \widehat{\delta}+2 \operatorname{Re}\left(\widehat{\mathrm{v}}^{*} \Pi_{11} \widehat{\delta}+\widehat{\mathrm{w}}^{*} \Pi_{12} \widehat{\delta}\right)\right] d \omega\right| \\
& \leq m_{11}\|\delta\|^{2}+2\|\delta\|\left(m_{11}\|v\|+m_{12}\|w\|\right) \\
& \leq c(\epsilon)\|\delta\|^{2}+\epsilon\left(\|v\|^{2}+\|w\|^{2}\right)
\end{aligned}
$$

for all $v, \delta \in \mathbf{L}_{2}^{l}[0, \infty), w \in \mathbf{L}_{2}^{m}[0, \infty)$. Note that (9) implies that

$$
\sigma_{\Pi}(G w, w) \leq-\epsilon|| w \|^{2}, \quad \forall w \in \mathbf{L}_{2}^{m}[0, \infty) .
$$

Let $\tau \in[0,1], w=\tau \Delta(v), v \in \mathbf{L}_{2}^{l}[0, \infty), \epsilon_{1}=\epsilon /(2+$ $\left.4\|G\|^{2}\right)$. Since $\tau \Delta$ satisfies the IQC defined by $\Pi$, we have

$$
\begin{aligned}
0 & \leq \sigma_{\Pi}(v, w)=\sigma_{\Pi}(G w, w)+\sigma_{\Pi}(v, w)-\sigma_{\Pi}(G w, w) \\
& \leq-\epsilon\|w\|^{2}+c\left(\epsilon_{1}\right)\|v-G w\|^{2}+\epsilon_{1}\left(\|w\|^{2}+\|v\|^{2}\right) \\
& \leq-\epsilon\|w\|^{2}+\left(c\left(\epsilon_{1}\right)+2 \epsilon_{1}\right)\|v-G w\|^{2} \\
& +\epsilon_{1}\left(\|w\|^{2}+2\|G w\|^{2}\right) \\
& \leq-\frac{\epsilon}{2}\|w\|^{2}+\left(c\left(\epsilon_{1}\right)+2 \epsilon_{1}\right)\|v-G w\|^{2} .
\end{aligned}
$$

Hence $\|w\| \leq$ const ||$v-G w \|$ and

$$
\|v\| \leq\|G w\|+\|v-G w\| \leq \text { const }\|v-\tau G \Delta(v)\| .
$$

Step 2: Show that if $(I-\tau G \Delta)^{-1}$ is bounded for some $\tau \in[0,1]$, then $(I-\nu G \Delta)^{-1}$ is bounded for any $\nu \in[0,1]$ with $|\tau-\nu|<\left(c_{0}\|G\| \cdot\|\Delta\|\right)^{-1}$. By the well-posedness assumption, the inverse $(I-\tau G \Delta)^{-1}$ is well defined on $\mathbf{L}_{2 \mathrm{e}}^{l}[0, \infty)$. Given $v \in \mathbf{L}_{2 \mathrm{e}}^{l}[0, \infty)$, define

$$
v_{T}=(I-\tau G \Delta)^{-1} P_{T}(v-\tau G \Delta(v)) .
$$

Then

$$
\begin{aligned}
& \left\|P_{T} v\right\|=\left\|P_{T} v_{T}\right\| \leq\left\|v_{T}\right\| \\
& \leq c_{0}\left\|v_{T}-\tau G \Delta\left(v_{T}\right)\right\|=c_{0}\left\|P_{T}(v-\tau G \Delta(v))\right\| \\
& \leq c_{0}\left\|P_{T}(v-\nu G \Delta(v))+(\nu-\tau) P_{T} G \Delta(v)\right\| \\
& \leq c_{0}\left\|P_{T}(v-\nu G \Delta(v))\right\|+c_{0}\|G \Delta\| \cdot|\tau-\nu| \cdot\left\|P_{T} v\right\| .
\end{aligned}
$$

Boundedness of $(I-\nu G \Delta)^{-1}$ follows, as $c_{0}\|G \Delta\| \cdot|\tau-\nu|<1$.

Step 3: Now, since $(I-\tau G \Delta)^{-1}$ is bounded for $\tau=0$, Step 2 shows that $(I-\tau G \Delta)^{-1}$ is bounded for $\tau$ smaller than $\left(c_{0}\|G\| \cdot\|\Delta\|\right)^{-1}$, then for $\tau<2\left(c_{0}\|G\| \cdot\|\Delta\|\right)^{-1}$, etc. By induction, it is bounded for all $\tau \in[0,1]$.

\section{APPLICATION TO ROBUSTNESS ANALYSIS}

In robustness analysis based on the feedback configuration illustrated in Fig. 1, it is natural to assume that $G(s)$ is known, and $\Delta$ describes the "trouble-making" (nonlinear, time-varying, or uncertain) components of the system.

First, we describe $\Delta$ as accurately as possible by IQC's. The class $\Pi_{\Delta}$ of all rational Hermitian matrix functions $\Pi$ that define a valid IQC for a given $\Delta$ is convex, and it is usually infinite-dimensional. For a large number of simple system components, a corresponding class $\boldsymbol{\Pi}_{\Delta}$ is readily available in the literature. In fact, IQC's are implicitly present in many results on robust/nonlinear/time-varying stability. A list of

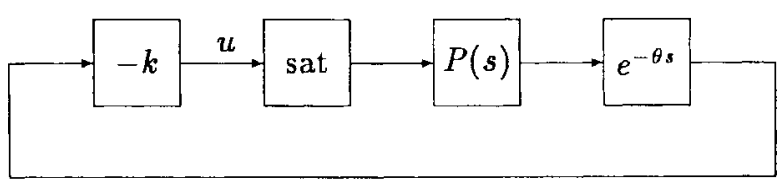

Fig. 2. System with saturation and delay.

such IQC's has been appended to this paper in Section VI. When $\Delta$ consists of a combination of several simple blocks, IQC's can be generated by convex combinations of constraints for the simpler components.

Next, we search for a matrix function $\Pi \in \Pi_{\Delta}$ that satisfies Theorem 1. The search for a suitable $\Pi$ can be carried out by numerical optimization, restricted to a finite-dimensional subset of $\Pi_{\Delta}$. Then, $\Pi$ can be written on the form

$$
\Pi(j \omega)=\sum_{q=1}^{q=q_{0}} x_{q} \Pi_{q}(j \omega)
$$

where $x_{q}$ are positive real parameters. Usually $\Pi_{q}$ and $G$ are proper rational functions with no poles on the imaginary axis, so there exists $n>0$, a Hurwitz matrix $A$ of size $n \times n$, a matrix $B$ of size $n \times m$, and a set of symmetric real matrices $M_{1}, \cdots, M_{q_{0}}$ of size $(n+m) \times(n+m)$, such that

$$
\begin{aligned}
{\left[\begin{array}{c}
G(j \omega) \\
I
\end{array}\right]^{*} \Pi_{q}(j \omega) } & {\left[\begin{array}{c}
G(j \omega) \\
I
\end{array}\right] } \\
& =\left[\begin{array}{c}
(j \omega I-A)^{-1} B \\
I
\end{array}\right]^{*} M_{q}\left[\begin{array}{c}
(j \omega I-A)^{-1} B \\
I
\end{array}\right]
\end{aligned}
$$

for all $q$. By application of the Kalman-Yakubovich-Popov lemma [19]-[21], it follows that (9) is equivalent to the existence of a symmetric $n \times n$ matrix $P=P^{T}$ such that

$$
\left[\begin{array}{cc}
P A+A^{T} P & P B \\
B^{T} P & 0
\end{array}\right]+\sum_{q=1}^{q=q_{0}} x_{q} M_{q}<0 .
$$

Hence the search for $x_{q}$ that produces a $\Pi$ satisfying (9) (i.e., proving the stability) takes the form of a convex optimization problem defined by a linear matrix inequality (LMI) in the variables $x_{q}, P$. Such problems can be solved very efficiently using the recently developed numerical algorithms based on interior point methods [9], [10].

\section{A. Example with Saturation and Delay}

Consider the following feedback system with control saturation and an uncertain delay:

$$
\begin{aligned}
& \dot{x}(t)=A x(t)+B \operatorname{sat}(\mathrm{u}(\mathrm{t})) \\
& u(t)=-k C x(t-\theta)
\end{aligned}
$$

where $k>0$ and $\theta \in\left[0, \theta_{0}\right]$ is an unknown constant

$$
P(s)=C(s I-A)^{-1} B=\frac{s^{2}}{s^{3}+2 s^{2}+2 s+1}
$$

is the transfer function of the controlled plant (see the Nyquist plot on Fig. 3), and

$$
\operatorname{sat}(u)=\left\{\begin{array}{cc}
u, & |u| \leq 1 \\
u /|u|, & |u| \geq 1
\end{array}\right.
$$

is the function that represents the saturation. The setup is illustrated in Fig. 2. 


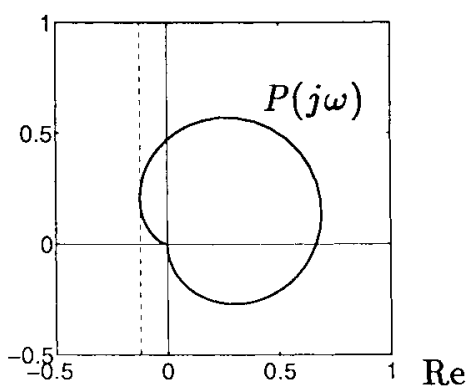

Fig. 3. Nyquist plot for $P(j \omega)$ (solid line).

Let us first consider stability analysis for the case of no delay. Then let $\Delta$ be the saturation, while $G(s)=-k P(s)$. Application of the circle criterion

$$
-k^{-1}<\min _{\omega} \operatorname{ReP}(\mathrm{j} \omega)
$$

gives stability for

$$
k<k_{\text {circ }} \approx 8.12
$$

(see dashed line in Fig. 3). This corresponds to a $\Pi_{\Delta}$ containing only the matrix

$$
\left[\begin{array}{cc}
0 & 1 \\
1 & -2
\end{array}\right]
$$

In the Popov criterion, $\Pi_{\Delta}$ consists of all linear combinations

$$
\left[\begin{array}{cc}
0 & 1 \\
1 & -2
\end{array}\right]+\eta\left[\begin{array}{cc}
0 & j \omega \\
-j \omega & 0
\end{array}\right]
$$

and the resulting inequality (9) gives the minor improvement

$$
\begin{aligned}
&-k^{-1}<\max _{\eta} \min _{\omega} \operatorname{Re}[(1+\mathrm{j} \omega \eta) \mathrm{P}(\mathrm{j} \omega)] \\
& k<k_{\text {Popov }} \approx 8.90 .
\end{aligned}
$$

A Popov plot is shown in Fig. 4.

Furthermore, because the saturation is monotone and odd, it is possible to apply a much stronger result, obtained by Zames and Falb [22]. By their statement, a sufficient condition for stability is the existence of a function $H \in \mathbf{R L}_{\infty}$ such that

$$
\begin{aligned}
0 & <\min _{\omega} \operatorname{Re}\left[\left(1+\mathrm{H}(\mathrm{j} \omega)^{*}\right)\left(\mathrm{P}(\mathrm{j} \omega)+\mathrm{k}^{-1}\right)\right] \\
H(j \omega) & =\int_{-\infty}^{\infty} e^{-j \omega t} h(t) d t \\
1 & \geq \int_{-\infty}^{\infty}|h(t)| d t .
\end{aligned}
$$

This extends the class of valid IQC's further, by allowing all matrix functions of the form

$$
\Pi(j \omega)=\left[\begin{array}{cc}
0 & 1+H(j \omega) \\
1+H(-j \omega) & -2(1+\operatorname{ReH}(j \omega))
\end{array}\right]
$$

where $H$ has an impulse response of $L_{1}$ norm no greater than one. For our problem, $H(j \omega)=-(1+j \omega)^{-1}$ gives for $\omega \in \mathbf{R}$ that

$$
\begin{aligned}
\operatorname{Re}[(1+H(-j \omega)) P(j \omega)] & \\
& =|1+H(-j \omega)|^{2} \operatorname{Re}\left(\frac{j \omega}{-\omega^{2}+j \omega+1}\right) \geq 0 .
\end{aligned}
$$

This shows that the feedback system is indeed stable for all $k>0$ and concludes the stability analysis in the undelayed case.

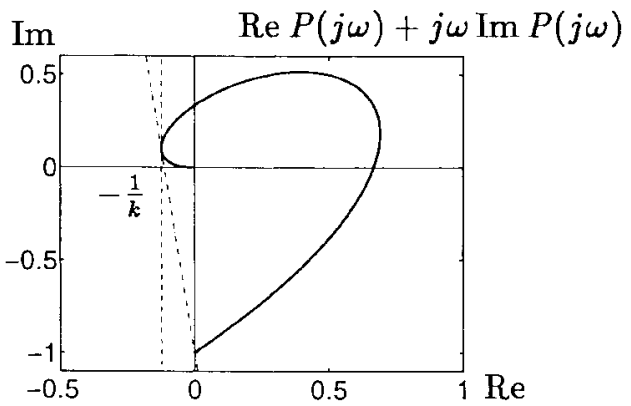

Fig. 4. Popov plot for $P(j \omega)$ with stabilizing gain $k$.

Considering also the delay uncertainty, the problem is to find a bound on the maximal stabilizing feedback gain for a given delay bound. A crude bound can be received directly from the small gain theorem, stating that, because of the gain bound $\left\|e^{-\theta s} \operatorname{sat}(\cdot)\right\|<1$, the feedback interconnection of $e^{-\theta s} \operatorname{sat}(\cdot)$ and $k P(s)$ is stable, provided that

$$
k<\|P\|_{\infty}^{-1} \approx 1.37 .
$$

Not surprisingly, this condition is conservative. For example, it does not utilize any bound on the delay. In order to do that, it is useful to generate more IQC's for the delay component. First, we rewrite (12) as a feedback interconnection on Fig. 1, with

$$
G(s)=\left[\begin{array}{cc}
-k P(s) & -k \\
P(s) & 0
\end{array}\right]
$$

and

$$
\Delta(v)(t)=\left[\begin{array}{c}
\operatorname{sat}\left(\mathrm{v}_{1}(\mathrm{t})\right) \\
v_{2}(t-\theta)-v_{2}(t)
\end{array}\right]
$$

With external signals $e, f$ as in (4), the equations are

$$
\begin{aligned}
\dot{x}(t) & =A x(t)+B w_{1}(t) \\
w_{1}(t) & =\operatorname{sat}\left[\mathrm{v}_{1}(\mathrm{t})\right]+\mathrm{e}_{1}(\mathrm{t}) \\
w_{2}(t) & =v_{2}(t-\theta)-v_{2}(t)+e_{2}(t) \\
v_{1}(t) & =-k C x(t)-k w_{2}(t)+f_{1}(t) \\
v_{2}(t) & =C x(t)+f_{2}(t)
\end{aligned}
$$

where $x(0)=0$ and $v_{2}(t-\theta)=0$ for $t<\theta$. One can see that (16) is equivalent to the equations from (12), disturbed by the "interconnection noise" $e, f$.

For the uncertain time delay, several types of IQC's are given in the list. Here we shall use a simple (and not complete) set of IQC's for the uncertain delay

$$
\widehat{w}_{2}(j \omega)=\left(e^{-j \theta \omega}-1\right) \widehat{v}_{2}(j \omega), \quad \theta \in\left[0, \theta_{0}\right]
$$

based on the bounds

$$
\begin{array}{r}
\left|\widehat{v}_{2}(j \omega)\right|^{2}-\left|\widehat{v}_{2}(j \omega)-\widehat{w}_{2}(j \omega)\right|^{2} \geq 0 \\
\psi_{0}\left(\theta_{0} \omega\right)\left|\widehat{v}_{2}(j \omega)\right|^{2}-\left|\widehat{w}_{2}(j \omega)\right|^{2} \geq 0
\end{array}
$$

where

$$
\psi_{0}(\omega)=\frac{\omega^{2}+0.08 \omega^{4}}{1+0.13 \omega^{2}+0.02 \omega^{4}}
$$

is chosen as a rational upper bound (see Fig. 5) of $\psi_{*}(j \omega)=\max _{\theta \in\left[0, \theta_{0}\right]}\left|e^{-j \omega \theta / \theta_{0}}-1\right|^{2}=\left\{\begin{array}{cc}4 \sin ^{2}(\omega / 2), & \omega<\pi \\ 4 & \omega \geq \pi .\end{array}\right.$ 


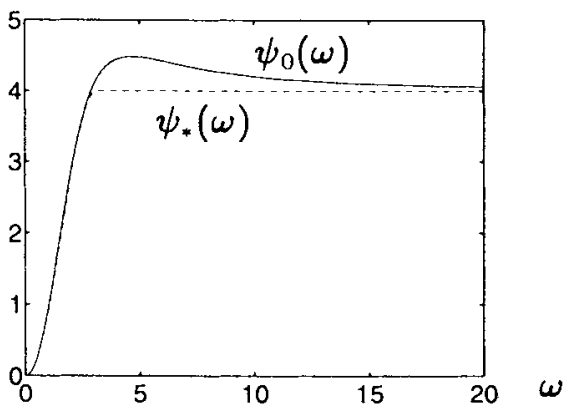

Fig. 5. Comparison of $\psi_{0}(\omega)$ and $\psi_{*}(j \omega)$.

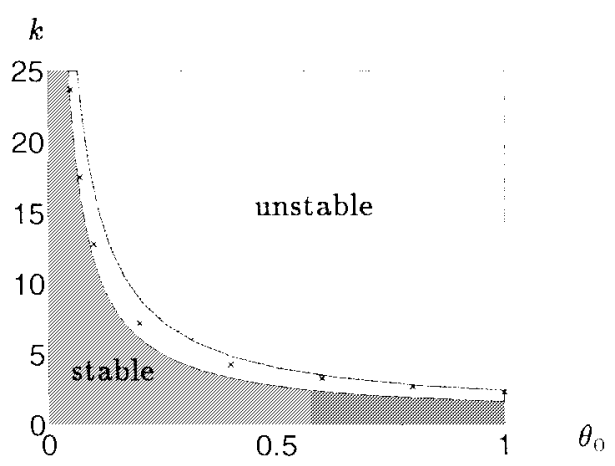

Fig. 6. Bounds on stabilizing gain $k$ versus delay uncertainty $\theta_{0}$.

By integrating the point-wise inequalities (17) with some nonnegative rational functions, one can obtain a huge set of IQC's valid for the uncertain delay. Using these in combination with some set of IQC's for the saturation nonlinearity, one can estimate the region of stability for the system given in (12). In Fig. 6, the "x"-marks denote parameter values for which stability has been proved using (17) for the delay and (15) with $H(s)=\tau(s+1)^{-1}, \tau \in[-1,1]$ for the saturation. The parameter $\tau$ was optimized by convex optimization. The guaranteed instability region was obtained analytically by considering the behavior of the system in the linear "unsaturated" region around the origin.

\section{HARD AND SOFT IQC'S}

As a rule, an IQC is an inequality describing correlation between the input and output signals of a causal block $\Delta$. Verifying an IQC can be viewed as a virtual experiment with the setup shown on Fig. 7, where $\Delta$ is the block tested for an IQC, $v$ is the test signal of finite energy, and $C(s)$ is a stable linear transfer matrix with two vector inputs, two vector outputs, and zero initial data. The blocks with $\int|\cdot|^{2} d t$ indicate calculation of the energy integral of the signal. We say that $\Delta$ satisfies the IQC described by the test setup if the energy of the second output of $C$ is always at least as large as the energy of the first output. Then the IQC can be represented in the form (1), where

$$
\Pi(j \omega)=C(j \omega)^{*}\left[\begin{array}{cc}
I & 0 \\
0 & -I
\end{array}\right] C(j \omega) .
$$

The most commonly used IQC is the one that expresses a gain bound on the operator $\Delta$. For example $C(s)=I$ corresponds to the bound $\|\Delta\| \leq 1$. The energy bounds have the

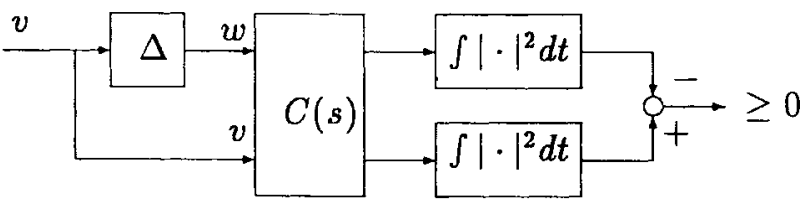

Fig. 7. Testing a block $\Delta$ for an IQC.

particular property that the energy difference until time $T$ will be nonnegative at any moment $T$, not just $T=\infty$. Such IQC's are called hard IQC's, in contrast to the more general soft IQC's, which need not hold for finite-time intervals. Some of the most simple IQC's are hard, but the "generic" ones are not.

Example: A simple example of a soft IQC is the one used in the Popov criterion. If $v, \dot{v} \in \mathbf{L}_{2}[0, \infty)$ and $v(0)=0$, then

$$
\int_{0}^{\infty} v \dot{v} d t=\lim _{T \rightarrow \infty}|v(T)|^{2}-|v(0)|^{2}=0 .
$$

Clearly, the integral on $[0, T]$ is nonnegative for every $T>0$, but not necessarily zero. Hence the IQC $\int_{0}^{\infty} v \dot{v} d t \geq 0$ is hard, but the IQC $\int_{0}^{\infty} v \dot{v} d t \leq 0$ is soft.

In the theory of absolute stability, the use of soft IQC's was often referred to as allowing "noncausal multipliers." While for scalar systems this was usually not a serious problem, the known conditions for applicability of noncausal multipliers were far too restrictive for multivariable systems. The formulation of Theorem 1 makes it possible (and easy) to use soft IQC's in a very general situation. For example, consider the following corollary.

Corollary 1 (Noncausal Multipliers): Assume that Condition i) of Theorem 1 is satisfied. If there exist some $M \in$ $\mathbf{R L}_{\infty}^{l \times m}$ and $\epsilon_{0}>0$ such that

$$
\begin{aligned}
\int_{-\infty}^{\infty} \operatorname{Re}\left(\widehat{\mathrm{v}}^{*} \mathrm{M} \widehat{\Delta v}\right) \mathrm{d} \omega & \geq 0, & & \text { for } v \in \mathbf{L}_{2}^{l}[0, \infty) \\
M^{*} G+G^{*} M & \leq-\epsilon_{0} G^{*} G, & & \text { on } j \mathbf{R}
\end{aligned}
$$

then the feedback interconnection of $G$ and $\Delta$ is input/output stable.

Proof: This is Theorem 1 with

$$
\Pi(j \omega)=\left[\begin{array}{cc}
\epsilon_{0} & M(j \omega) \\
M(j \omega)^{*} & -\epsilon_{0} /\|\Delta\|^{2}
\end{array}\right]
$$

and $\epsilon=\epsilon_{0} /\|\Delta\|^{2}$. The IQC for $\Delta$ follows as

$$
\begin{aligned}
& \int_{-\infty}^{\infty}\left[\frac{\widehat{v}(j \omega)}{\Delta v(j \omega)}\right]^{*} \Pi(j \omega)[\widehat{\widehat{v}(j \omega)}[\hat{\Delta v}(j \omega)] d \omega \\
& =\int_{-\infty}^{\infty} \operatorname{Re}\left(\widehat{\mathrm{v}}^{*} \mathrm{M} \widehat{\Delta v}+\epsilon_{0}|\mathrm{v}|^{2}-\epsilon_{0}|\Delta \mathrm{v}|^{2} /\|\Delta\|^{2}\right) \mathrm{d} \omega \geq 0 .
\end{aligned}
$$

For multivariable systems, the above conditions on $M$ are much weaker than factorizability as $M=M_{-} M_{+}$, with $M_{+}, M_{+}{ }^{-1}, M_{-}{ }^{*},\left(M_{-}{ }^{*}\right)^{-1}$ all being stable, which is required, for example, in [22] and [6]. The price paid for this in Theorem 1 is the very mild assumption that the feedback loop is well posed not only for $\tau=1$, but for all $\tau \in[0,1]$.

Another example is provided by the classical Popov criterion. 


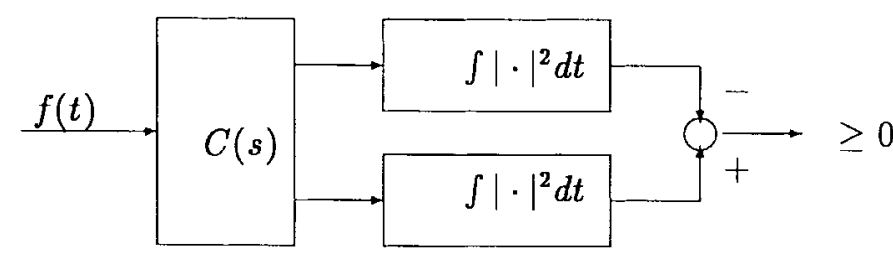

Fig. 8. Testing a signal $f$ for an IQC.

Corollary 2 (Popov Criterion): Assume that $\phi: \mathbf{R} \rightarrow \mathbf{R}$ is such that $0 \leq \sigma \phi(\sigma) \leq$ const $\cdot \sigma^{2}$ for $\sigma \in \mathbf{R}$. Let $H(s)=C(s I-A)^{-1} B$, where $A$ is Hurwitz. Assume that the system

$$
\dot{x}(t)=A x(t)+B \tau \phi(C x(t))+f(t)
$$

has unique solution on $[0, \infty)$ for any $\tau \in[0,1]$ and for any square summable $f$. If for some $q \in \mathbf{R}$

$$
\inf _{\omega>0} \operatorname{Re}[(1+j \omega q) H(j \omega)]>0
$$

then (19) with $\tau=1$ is exponentially stable.

Proof: For $q \in \mathbf{R}$ and a differentiable $u \in \mathbf{L}_{2}^{l}[0, \infty)$, we have the soft IQC

$$
\int_{0}^{\infty}(u+q \dot{u}) \phi(u) d t \geq q\left[\int_{0}^{u(t)} \phi(\sigma) d \sigma\right]_{0}^{\infty}=0 .
$$

Application of Corollary 1 with

$$
\begin{aligned}
G(s) & =(s+1) H(s) \\
(\Delta v)(t) & =\phi\left(\int_{0}^{t} e^{-\tau} v(\tau) d \tau\right) \\
M(s) & =(1+q s) /(s+1)
\end{aligned}
$$

shows that the conditions of Proposition 8 hold, which ensures the exponential stability.

IQC's can be used to describe an external signal (noise or a reference) entering the system. The "virtual experiment" setup for a signal $f$ is shown on Fig. 8. The setup clearly shows the "spectral analysis" nature of IQC's describing the signals. Mathematically, the resulting IQC has the form

$$
\int_{-\infty}^{\infty} \hat{f}(j \omega)^{*} \Pi(j \omega) \hat{f}(j \omega) d \omega \geq 0
$$

where $\Pi$ is given by (18).

Performance analysis of systems can be made with both interior blocks and external signals described in terms of IQC's.

\section{IQC'S AND QUADRATIC STABILITY}

There is a close relationship between quadratic stability and stability analysis based on IQC's. As a rule, if a system is quadratically stable, then its stability can also be proved by using a simple IQC. Conversely, a system that can be proved to be stable via IQC's always has a quadratic Lyapunov function in some generalized sense. However, to actually present this Lyapunov function, one has to extend the state space of the system (by adding the states of $C(s)$ from Fig. 7). Even then, in the case of soft IQC's, the Lyapunov function does not need to be sign-definite and may not decrease monotonically along the system trajectories. In any case, use of IQC's replaces the "blind" search for a quadratic Lyapunov function, which is typical for the quadratic stability, by a more sophisticated search. In general, for example in the case of so-called "parameter-dependent" Lyapunov functions, the relationship with the IQC type analysis has yet to be clarified.

Below we formulate and prove a result on the relationship between a simple version of quadratic stability and IQC's. Let $\mathcal{D}$ be a polytope of $m \times l$ matrices $\Delta$, containing the zero matrix $\Delta=0$. Let $\Delta_{1}, \cdots, \Delta_{N}$ be the extremal points of $\mathcal{D}$. Consider the system of differential equations

$$
\dot{x}(t)=(A+B \Delta(t) C) x(t), \quad \Delta(t) \in \mathcal{D}
$$

where $A, B, C$ are given matrices of appropriate size, and $A$ is a Hurwitz $n \times n$ matrix. (The most often considered case of (21) is obtained when $m=l$ and $\mathcal{D}$ is the set of all diagonal matrices with the norm not exceeding 1 . Then $N=2^{m}$, and $\Delta_{i}$ are the diagonal matrices with \pm 1 on the diagonal.) The system is called stable if $x(t) \rightarrow 0$ for any solution of $(21)$, where $\Delta(\cdot)$ is a measurable function and $\Delta(t) \in \mathcal{D}$ for all $t$. There are no efficient general conditions that are both necessary and sufficient for the stability of (21). Instead, we will be concerned with stability conditions that are only sufficient.

System (21) is called quadratically stable if there exists a matrix $P=P^{T}$ such that

$$
P\left(A+B \Delta_{i} C\right)+\left(A+B \Delta_{i} C\right)^{T} P<0, \quad \forall i .
$$

Note that since $0 \in \mathcal{D}$ and $A$ is a Hurwitz matrix, this condition implies that $P>0$. It follows that $V(x)=x^{T} P x$ is a Lyapunov function for (21) in the sense that $V$ is positive definite and $d V(x(t)) / d t$ is negative definite on the trajectories. Quadratic stability is a sufficient condition for stability of the system, and (22) can be solved efficiently with respect to $P=P^{T}$ as a system of linear matrix inequalities.

An IQC-based approach to stability analysis of (21) can be formulated as follows. Note that stability of (21) is equivalent to stability of the feedback interconnection (4), where $G$ is the linear time-invariant operator with transfer function $G(s)=C(s I-A)^{-1} B$, and $\Delta$ is the operator of multiplication by $\Delta(t) \in \mathcal{D}$. One can apply Theorem 1 , using the fact that $\Delta$ satisfies the IQC's given by the constant multiplier matrix

$$
\Pi(j \omega)=\left[\begin{array}{cc}
Q & S \\
S^{T} & R
\end{array}\right]
$$

where $Q=Q^{T}, R=R^{T}, S$ are real matrices such that

$$
Q+S \Delta+\Delta^{T} S^{T}+\Delta^{T} R \Delta>0, \quad \forall \Delta \in \mathcal{D} .
$$

For a fixed matrix $\Pi$ satisfying (23), a sufficient condition of stability given by Theorem 1 is

$$
\left[\begin{array}{c}
G(j \omega) \\
I
\end{array}\right]^{*} \Pi\left[\begin{array}{c}
G(j \omega) \\
I
\end{array}\right]<0, \quad \forall \omega \in \mathbf{R} \cup\{\infty\}
$$

which is equivalent (by the Kalman-Yakubovich-Popov lemma) to the existence of a matrix $P=P^{T}$ such that

$$
\left[\begin{array}{cc}
P A+A^{T} P+C^{T} Q C & P B+C^{T} S \\
B^{T} P+S^{T} C & R
\end{array}\right]<0 .
$$


For an indefinite matrix $R$, (23) may be difficult to verify. However, (24) yields $R<0$. In that case, it is sufficient to check (23) at the vertices $\Delta=\Delta_{i}$ of $\mathcal{D}$ only, i.e., (23) can be replaced by

$$
Q+S \Delta_{i}+\Delta_{i}^{T} S^{T}+\Delta_{i}^{T} R \Delta_{i}>0, \quad \forall i .
$$

It is easy to see that the existence of the matrices $P=$ $P^{T}, Q=Q^{T}, S, R=R^{T}$, such that (24), (25) hold, is a sufficient condition of stability of (21).

Now we have the two seemingly different conditions for stability of (21), both expressed in terms of systems of LMI's: quadratic stability (22) and IQC-stability (24), (25). The first condition has $n(n+1) / 2$-free variables (the components of the matrix $\left.P=P^{T}\right)$, while the second condition has $n(n+1) / 2+(n+m)(n+m+1) / 2$-free variables. However, the advantage of using the IQC condition is that the overall "size" of the corresponding LMI is $n+m+N l$, while the total "size" of the condition for quadratic stability is $N n$. If $N$ is a large number and $n$ is significantly larger than $l$ and $m$, a modest (about two times) increase of the number of free variables in (24), (25) results in a significant (about $n / l$ times) decrease in the size of the corresponding LMI. The following result shows that the two sufficient conditions of stability (24), (25) and (22) are equivalent from the theoretical point of view.

Theorem 2: Assume that $A$ is a Hurwitz matrix and that zero belongs to the convex hull of matrices $\Delta_{1}, \cdots, \Delta_{N}$. Then, a given symmetric matrix $P$ solves the system of LMI's (22), if and only if $P$ together with some matrices $Q=Q^{T}, R=R^{T}, S$ solve (24) and (25).

Proof: The sufficiency is straightforward: multiplying (24) by $\left[\begin{array}{ll}I & C^{T} \Delta_{i}^{T}\end{array}\right]$ from the left, and by $\left[\begin{array}{ll}I & C^{T} \Delta_{i}^{T}\end{array}\right]^{T}$ from the right yields

$$
\begin{aligned}
P\left(A+B \Delta_{i} C\right)+ & \left(A+B \Delta_{i} C\right)^{T} P \\
+ & C^{T}\left(Q+S \Delta_{i}+\Delta_{i}^{T} S^{T}+\Delta_{i}^{T} R \Delta_{i}\right) C<0
\end{aligned}
$$

which implies (22) because of the inequality in (25).

To prove the necessity, let $P=P^{T}$ satisfy (22). Let $\sigma_{0}: \mathbf{R}^{n} \times \mathbf{R}^{m} \rightarrow \mathbf{R}$ be the quadratic form

$$
\sigma_{0}(x, \xi)=-\epsilon\left(|x|^{2}+|\xi|^{2}\right)-2 x^{T} P(A x+B \xi)
$$

where $\epsilon>0$ is a small parameter. Define $\sigma: \mathbf{R}^{l} \times \mathbf{R}^{m} \rightarrow \mathbf{R}$ by

$$
\sigma(y, \xi)=\inf \left\{\sigma_{0}(x, \xi): C x=y\right\}
$$

where the infimum is taken over all $x \in \mathbf{R}^{n}$ such that $C x=y$. Since the zero matrix belongs to the convex hull of $\mathcal{D}$, (22) implies that $P A+A^{T} P<0$. Hence, for a sufficiently small $\epsilon>0, \sigma$ is strictly convex in the first argument, and a finite minimum in (26) exists. Moreover, since $\sigma_{0}$ is a quadratic form, the same is true for $\sigma$, and the matrices $Q, R, S$ can be introduced by

$$
\sigma(y, \xi)=y^{T} Q y+2 y^{T} S \xi+\xi^{T} R \xi .
$$

Let us show that the inequalities (24), (25) are satisfied. First, by (22), for any $y$ we have

$$
\begin{aligned}
& y^{T}\left(Q+S \Delta_{i}+\Delta_{i}^{T} S^{T}+\Delta_{i}^{T} R \Delta_{i}\right) y \\
&= \sigma\left(y, \Delta_{i} y\right) \\
&= \inf \left\{\sigma_{0}\left(x, \Delta_{i} C x\right): C x=y\right\} \\
&= \inf \left\{-x^{T}\left(P\left(A+B \Delta_{i} C\right)+\left(A+B \Delta_{i} C\right)^{T} P\right) x\right. \\
&\left.\quad-\epsilon\left(|x|^{2}+\left|\Delta_{i} C x\right|^{2}\right): C x=y\right\} \\
& \geq \epsilon_{1}|y|^{2}
\end{aligned}
$$

(provided that $\epsilon$ and $\epsilon_{1}$ are sufficiently small). Hence (25) holds. Similarly, for any $x, \xi$ we have

$$
\begin{aligned}
& x^{T} P(A x+B \xi)+\sigma(C x, \xi) \\
& \quad=x^{T} P(A x+B \xi)+\inf \left\{\sigma_{0}\left(x_{1}, \xi\right): C x_{1}=C x\right\} \\
& \quad \leq x^{T} P(A x+B \xi)-\epsilon\left(|x|^{2}+|\xi|^{2}\right)-x^{T} P(A x+B \xi) \\
& \quad \leq-\epsilon\left(|x|^{2}+|\xi|^{2}\right)
\end{aligned}
$$

and hence (24) holds, since the matrix in (24) is the matrix of the quadratic form $x^{T} P(A x+B \xi)+\sigma(C x, \xi)$.

\section{A LIST OF IQC'S}

The collection of IQC's presented in this section is far from being complete. However, the authors hope it will support the idea that many important properties of basic system interconnections used in stability analysis can be characterized by IQC's.

\section{A. Uncertain Linear Time-Invariant Dynamics}

Let $\Delta$ be any linear time-invariant operator with gain $\left(H_{\infty}\right.$ norm) less than one. Then $\Delta$ satisfies all IQC's of the form

$$
\left[\begin{array}{cc}
x(j \omega) I & 0 \\
0 & -x(j \omega) I
\end{array}\right]
$$

where $x(j \omega) \geq 0$ is a bounded measurable function.

\section{B. Constant Real Scalar}

If $\Delta$ is defined by multiplication with a real number of absolute value $\leq 1$, then it satisfies all IQC's defined by matrix functions of the form

$$
\left[\begin{array}{cc}
X(j \omega) & Y(j \omega) \\
Y(j \omega)^{*} & -X(j \omega)
\end{array}\right]
$$

where $X(j \omega)=X(j \omega)^{*} \geq 0$ and $Y(j \omega)=-Y(j \omega)^{*}$ are bounded and measurable matrix functions.

This IQC and the previous one are the basis for standard upper bounds for structured singular values [23], [24].

\section{Time-Varying Real Scalar}

Let $\Delta$ be defined by multiplication in the time-domain with a scalar function $\delta \in \mathbf{L}_{\infty}$ with $\|\delta\|_{\infty} \leq 1$. Then $\Delta$ satisfies IQC's defined by a matrix of the form

$$
\left[\begin{array}{cc}
X & Y \\
Y^{T} & -X
\end{array}\right]
$$

where $X=X^{T} \geq 0$ and $Y=-Y^{T}$ are real matrices. 


\section{Coefficients from a Polytope}

Let $\Delta$ be defined by multiplication in the time-domain with a measurable matrix $\Delta(\cdot)$ such that $\Delta(t) \in \mathcal{D}$ for any $t$, where $\mathcal{D}$ is a polytope of matrices with the extremal points (vertices) $\Delta_{1}, \cdots, \Delta_{N} . \Delta$ satisfies the IQC's given by the constant weight matrices

$$
\Pi(j \omega)=\left[\begin{array}{cc}
Q & F \\
F^{T} & R
\end{array}\right]
$$

where $Q=Q^{T}, F, R=R^{T}$ are real matrices such that $R \leq 0$, and

$$
Q+F \Delta_{i}+\Delta_{i}^{T} F^{T}+\Delta_{i}^{T} R \Delta_{i}>0, \quad \forall i .
$$

This IQC corresponds to quadratic stability and was studied in Section V.

\section{E. Periodic Real Scalar}

Let $\Delta$ be defined by multiplication in the time-domain with a periodic scalar function $\delta \in \mathbf{L}_{\infty}$ with $\|\delta\|_{\infty} \leq 1$ and period $T$. Then $\Delta$ satisfies IQC's defined by (27), where $X$ and $Y$ are bounded, measurable matrix functions satisfying

$$
\begin{aligned}
& X(j \omega)=X(j(\omega+2 \pi / T))=X(j \omega)^{*} \geq 0 \\
& Y(j \omega)=Y(j(\omega+2 \pi / T))=-Y(j \omega)^{*} .
\end{aligned}
$$

This set of IQC's gives the result by Willems on stability of systems with uncertain periodic gains [19].

\section{F. Multiplication by a Harmonic Oscillation}

If $(\Delta v)(t)=v(t) \cos \left(\omega_{0} t\right)$, then $\Delta$ satisfies the IQC's defined by

$$
\Pi(j \omega)=\left[\begin{array}{cc}
X\left(j \omega-j \omega_{0}\right)+X\left(j \omega+j \omega_{0}\right) & 0 \\
0 & -2 X(j \omega)
\end{array}\right]
$$

where $X(j \omega)=X(j \omega)^{*} \geq 0$ is any bounded matrixvalued rational function. Multiplication by a more complicated (almost periodic) function can be represented as a sum of several multiplications by a harmonic oscillation with the IQC's derived for each of them separately. For example

$v(t)\left\{a_{1} \cos \left(\omega_{1} t\right)+a_{2} \cos \left(\omega_{2} t\right)\right\}=a_{1}\left(\Delta_{1} v\right)(t)+a_{2}\left(\Delta_{2} v\right)(t)$

where

$$
\begin{aligned}
& \left(\Delta_{1} v\right)(t)=v(t) \cos \left(\omega_{1} t\right) \\
& \left(\Delta_{2} v\right)(t)=v(t) \cos \left(\omega_{2} t\right) .
\end{aligned}
$$

\section{G. Slowly Time-Varying Real Scalar}

Here $\Delta$ is the operator of multiplication by a slowly timevarying scalar, $\Delta v=\delta(t) v(t)$, where $|\delta(t)| \leq 1,|\dot{\delta}(t)| \leq d$. Since the 1960's, various IQC's have been discovered that hold for such time variations; see, for example, [25]-[27].

Here we describe a simple but representative family of IQC's describing the redistribution of energy among frequencies, caused by the multiplication by a slowly time-varying coefficient. For any transfer matrix

$$
H(s)=H_{0}+\int_{-\infty}^{+\infty} e^{-t s} h(t) d t
$$

where $h(\cdot) \in L_{1}^{n \times m}(-\infty,+\infty)$ and $H_{0}$ is a constant, let $\phi(H, d)$ be an upper bound of the norm of the commutator $\Delta \circ H-H \circ \Delta$, for example

$$
\phi(H, d)=\int_{-\infty}^{+\infty}\|h(t)\| \min \{2, d|t|\} d t .
$$

The following weighting matrices then define valid IQC's:

$$
\Pi=\left[\begin{array}{cc}
(1+\rho)\left\{H^{*} H+\frac{\phi(H, d)^{2}}{\rho} I_{m}\right\} & 0 \\
0 & -H^{*} H
\end{array}\right]
$$

where $\rho>0$ is a parameter, and $H$ is a causal transfer function $(h(t)=0$ for $t<0)$. Another set of IQC's is given by

$$
\Pi=\left[\begin{array}{cc}
\phi(H, d) I & H \\
H^{*} & 0
\end{array}\right]
$$

where $H$ is skew-Hermitian along the imaginary axis (i.e., $\left.H(j \omega)=-H(j \omega)^{*}\right)$ but not necessarily causal. Since

$$
\phi(H, d)=O(d) \text { as } d \rightarrow 0
$$

whenever $\|h(t)\|=O\left(t^{-2-\epsilon}\right)$, the constraints used in the " $\mu$ " case (multiplication by a constant gain $\delta \in[-1,1]$ ) can be recovered from (28) and (29) as $d \rightarrow 0$. Similarly, the "timevarying real scalar" IQC's will be recovered as $d \rightarrow \infty$ by using constant transfer matrices $H(s)=H_{0}$.

In [28] and [29], IQC's are instead derived for uncertain time-varying parameters with bounds on the support of the Fourier transform $\hat{\delta}$. Slow variation then means that $\hat{\delta}(j \omega)$ is zero except for $\omega$ in some small interval $[-a, a]$.

\section{H. Delay}

The uncertain bounded delay operator $(\Delta v)(t)=v(t-\theta)=$ $w(t)$, where $\theta \in\left[0, \theta_{0}\right]$, satisfies the "point-wise" quadratic constraints in the frequency domain

$$
\begin{gathered}
|\widehat{w}(j \omega)|^{2}=|\widehat{v}(j \omega)|^{2} \\
\psi_{1}\left(\omega_{*}\right)\left(\left|j \omega_{*} \widehat{w}(j \omega)+\widehat{v}(j \omega)\right|^{2}-\left(1+\omega_{*}^{2}\right)|\widehat{v}(j \omega)|^{2}\right) \\
\geq \psi_{2}\left(j \omega_{*}\right)|\widehat{v}(j \omega)-\widehat{w}(j \omega)|^{2}
\end{gathered}
$$

where $\omega_{*}=\omega \theta_{0} / 2$, and $\psi_{1,2}$ are the functions defined by

$$
\begin{aligned}
& \psi_{1}(\omega)= \begin{cases}\frac{\sin \omega}{\omega}, & |\omega| \leq \pi \\
0, & |\omega|>\pi\end{cases} \\
& \psi_{2}(\omega)= \begin{cases}\cos \omega, & |\omega| \leq \pi \\
0, & |\omega|>\pi\end{cases}
\end{aligned}
$$

Note that (31) is just a sector inequality for the relation between $\widehat{v}(j \omega)-\widehat{w}(j \omega)$ and $j(\widehat{v}(j \omega)+\widehat{w}(j \omega))$

$$
j(\widehat{v}(j \omega)+\widehat{w}(j \omega))=\frac{\cos (\omega \theta / 2)}{\sin (\omega \theta / 2)}(\widehat{v}(j \omega)-\widehat{w}(j \omega)) .
$$

Multiplying (30) by any rational function and integrating over the imaginary axis yields a set of IQC's for the delay. Unfortunately, these IQC's do not utilize the bound on the delay. To improve the IQC-description, one can multiply (31) by any nonnegative weight function and integrate over the imaginary axis. The resulting IQC's, however, will have nonrational weight matrices $\Pi(\cdot)$. Instead, one should use a 
rational upper bound $\psi_{1+}$ of $\psi_{1}$ and rational lower bounds $\psi_{1-}$ and $\psi_{2-}$ of $\psi_{1}$ and $\psi_{2}$, respectively. For example, a reasonably good approximation is given by

$$
\begin{aligned}
& \psi_{1+}=\frac{\left(1-0.0646 \omega^{2}\right)^{2}}{1+0.038 \omega^{2}+0.0001 \omega^{4}+0.00085 \omega^{6}} \\
& \psi_{1-}=\frac{1-\omega^{2} / \pi^{2}}{1+\left(1 / 6-1 / \pi^{2}\right) \omega^{2}+\left(2 / \pi^{4}-1 / 6 \pi^{2}\right) \omega^{4}} \\
& \psi_{2-}=\frac{1-0.4073 \omega^{2}}{1+0.0927 \omega^{2}+0.0085 \omega^{4}} .
\end{aligned}
$$

Then the point-wise inequality (31) holds with $\psi_{2}$ replaced by $\psi_{2-}$, and with $\psi_{1}$ replaced by $\psi_{1+}$ (the upper bound for the $\left|j \omega_{*} \widehat{w}+\widehat{v}\right|^{2}$ multiplier, the lower bound for the $|\widehat{v}|^{2}$ multiplier), respectively, and can be integrated with a nonnegative rational weight function to get rational IQC's utilizing the upper bound on the delay.

A simpler, but less informative, set of IQC's is defined for $(\Delta v)(t)=v(t-\theta)-v(t), \theta \leq \theta_{0}$, by

$$
\left[\begin{array}{cc}
\tau(j \omega) \psi_{0}\left(\omega \theta_{0}\right) & 0 \\
0 & -\tau(j \omega)
\end{array}\right]
$$

where $\tau(\cdot)$ is any nonnegative rational weighting function, and $\psi_{0}(\omega)$ is any rational upper bound of

$$
\psi_{*}(\omega)=\max _{\theta \in\left[0, \theta_{0}\right]}\left|e^{-j \omega \theta / \theta_{0}}-1\right|^{2}=\left\{\begin{array}{cc}
4 \sin ^{2}(\omega / 2), & \omega<\pi \\
4 & \omega \geq \pi
\end{array}\right.
$$

for example

$$
\psi_{0}(\omega)=\frac{\omega^{2}+0.08 \omega^{4}}{1+0.13 \omega^{2}+0.02 \omega^{4}}
$$

\section{Memoryless Nonlinearity in a Sector}

If $(\Delta v)(t)=\phi(v(t), t)$, where $\phi: \mathbf{R} \times \mathbf{R} \rightarrow \mathbf{R}$ is a function such that

$$
\alpha v^{2} \leq \phi(v, t) v \leq \beta v^{2}, \quad \forall v \in \mathbf{R}, t \geq 0
$$

then obviously the IQC with

$$
\Pi(j \omega)=\left[\begin{array}{cc}
-2 \alpha \beta & \alpha+\beta \\
\alpha+\beta & -2
\end{array}\right]
$$

holds.

\section{J. The "Popov" IQC}

If $w(t)=(\Delta v)(t)=\phi(v(t))$, where $\phi: \mathbf{R} \rightarrow \mathbf{R}$ is a continuous function, $v(0)=0$, and both $w(\cdot)$ and $\dot{v}(\cdot)$ are square summable, then

$$
\int_{0}^{\infty} \dot{v}(t) w(t) d t=0
$$

In the frequency domain, this looks like an IQC with

$$
\Pi(j \omega)= \pm\left[\begin{array}{cc}
0 & j \omega \\
-j \omega & 0
\end{array}\right] .
$$

However, this is not a "proper" IQC, because $\Pi(\cdot)$ is not bounded on the imaginary axis. To fix the problem, consider $\Delta_{1}=\Delta \circ \frac{1}{s+1}$ instead of $\Delta$, i.e., $w(t)=\left(\Delta_{1} f\right)(t)=\phi(v(t))$, where $\dot{v}(t)=-v(t)+f(t), v(0)=0$. Now, $\Delta_{1}$ satisfies the IQC with

$$
\Pi(j \omega)= \pm\left[\begin{array}{cc}
0 & \frac{j \omega}{1+j \omega} \\
-\frac{j \omega}{1-j \omega} & 0
\end{array}\right] .
$$

Together with the IQC for a memoryless nonlinearity in a sector, this IQC yields the well-known Popov criterion.

\section{K. Monotonic Odd Nonlinearity}

Suppose $\Delta$ operates on scalar signals according to the nonlinear map $(\Delta v)(t)=\delta(v(t))$, where $\delta$ is an odd function on $\mathbf{R}$ such that $\dot{\delta}(x) \in[0, k]$ for some constant $k$. Then $\Delta$ satisfies the IQC's defined by

$$
\left[\begin{array}{cc}
0 & 1+H(j \omega) \\
1+H(-j \omega) & -(2+2 \operatorname{ReH}(\mathrm{j} \omega)) / \mathrm{k}
\end{array}\right]
$$

where $H \in \mathbf{R L}_{\infty}$ is arbitrary except that the $L_{1}$-norm of its impulse response is no larger than one [22].

\section{IQC's for Signals}

Performance of a linear control system is often measured in terms of disturbance attenuation. An important issue is then the definition of the set of expected external signals. Here again, IQC's can be used as a flexible tool, for example to specify bounds on auto correlation, frequency distribution, or even to characterize a given finite set of signals. Then, the information given by the IQC's can be used in the performance analysis, along the lines discussed in [49] and [30].

\section{IQC's from Robust Performance}

One of the most appealing features of IQC's is their ability to widen the field of application of already existing results. This means that almost any robustness result derived by some method (possibly unrelated to the IQC techniques) for a special class of systems can be translated into an IQC.

As an example of such a "translation," consider the feedback interconnection of a particular linear time-invariant system $G_{0}=G_{0}(s)$ with an "uncertain" block $\Delta$

$$
v=G_{0} w+f, \quad w=\Delta(v)
$$

where $f$ is the external disturbance. Assume that stability of this interconnection (i.e., the invertibility of the operator $I-G_{0} \Delta$ ) is already proved, and, moreover, an upper bound on the induced $L_{2}$ gain "from $f$ to $v$ " ("robust performance") is known; $\|v\|^{2} \leq d\|f\|^{2}$ for any square summable $f, v$ satisfying (32). Then, since for any square summable $v$ there exists a square summable $f=v-G_{0} \Delta(v)$ satisfying (32), the block $\Delta$ satisfies the IQC given by

$$
\Pi(j \omega)=\left[\begin{array}{cc}
d-1 & -d G_{0}(j \omega) \\
-d G_{0}(-j \omega) & d\left|G_{0}(j \omega)\right|^{2}
\end{array}\right] .
$$

This IQC implies stability of system (32) via Theorem 1 but can also be used in the analysis of systems with additional feedback blocks, as well as with different nominal transfer functions.

For example, consider the uncertain block $\Delta$ which represents multiplication of a scalar input by a scalar time-varying 
coefficient $k=k(t)$, such that $k(t) \in[-1,1]$. There is one obvious IQC for this block, stating that the $L_{2}$-induced norm of $\Delta$ is not greater than one. Let us show how additional nontrivial IQC's can be derived, based on a particular robust performance result. Consider the feedback interconnection of $\Delta$ with a given linear time-invariant block with a stable transfer function $G_{0}(s)=C(s I-A)^{-1} B$. This is the case of a system with one uncertain fast time-varying parameter $k=k(t), k(t) \in[-1,1]$

$$
\dot{x}(t)=A x(t)+B k(t)(C x(t)+f(t))
$$

where $A, B, C$ are given constant matrices, $A$ is a Hurwitz matrix, $f(\cdot)$ is the external disturbance. It is known that, for this system, the norm bound $\|v\|^{2} \leq\|\Delta(v)\|^{2}$, yields the circle stability criterion $\left|G_{0}(j \omega)\right|<1$, which gives only sufficient conditions of stability. Nevertheless, for a large class of transfer functions $G_{0}(s)$, not satisfying the circle criterion, (34) is robustly stable. A proof of such stability usually involves using a nonquadratic Lyapunov function $V=V(x)$, and provides an upper bound $d$ of the worst-case $L_{2}$-induced gain "from $v$ to $y=C x+v$ ". This upper bound, in turn, yields the IQC given by (33), describing the uncertain block $\Delta$. The fact that stability of (34) can be proved from this new IQC, but not from the simple norm bound $\|\Delta(v)\|^{2} \leq\|v\|^{2}$, shows that the new IQC indeed carries additional information about $\Delta$.

\section{CONCLUSIONS}

The objective of the paper was to give an overview of the IQC-based stability analysis, featuring a basic stability theorem and a list of the most important IQC's. Depending on the application, several modifications of the basic framework can be used, providing more flexibility in the analysis as well as open problems for future research.

Unconditional and Conditional IQC's: In this paper, an operator $\Delta$ was said to satisfy an IQC if (1) was satisfied for any $v, w \in \mathbf{L}_{2}[0, \infty)$ such that $w=\Delta(v)$. Such an IQC can be called "unconditional," because it does not depend on the environment in which the block $\Delta$ is being used. Using such IQC's is easy and convenient, in particular because they can be derived independently of the system setup (or be found in the literature). Sometimes, however, unconditional IQC's lead to unjustified conservatism in the analysis. Consider, for simplicity, the system

$$
\left\{\begin{array}{l}
\dot{x}(t)=A x(t)+B w(t) \\
v(t)=C x(t) \\
w(t)=\Delta(v(t))
\end{array}\right.
$$

where $\Delta$ is a given nonlinear function. Here the set of possible functions $y(\cdot)$ is relatively small [it is parameterized by the initial data parameter $x(0)$ ]. Therefore, it may be an "overkill" to consider the relation between $v$ and $w=\Delta(v)$ for all square summable $v$. It should be sufficient to look only at those $v$ that may be produced by (35). For example, if $\Delta(v)=\operatorname{sat}(\mathrm{v})$, then the "unconditional" IQC (2) with

$$
\sigma\left(x_{c}, v, w\right)=|v-0.5 w|^{2}-\epsilon|w|^{2}
$$

does not hold for any $\epsilon>0$. However, it holds (with sufficiently small $\epsilon$ ) as a conditional IQC whenever $A+0.5 B C$ is a nonsingular matrix.

Average IQC's: The “average" IQC's are especially useful in their conditional form when one is working with stochastic system models. These IQC's are defined by replacing (2) with

$$
\limsup _{T \rightarrow \infty} \frac{1}{T} \int_{0}^{T} \sigma\left(x_{c}(t), y(t), \xi(t)\right) d t \geq 0 .
$$

Incremental IQC's: For nonlinear systems, as a rule, the standard IQC's (1) are good only for showing that the signals within the system are "small" (are square summable, tend to zero, etc.) However, many interesting questions, for example the study of the existence and properties of a globally attractive periodic response to any periodic input, require deeper information about the system. This can be supplied by incremental IQC's. An unconditional incremental IQC describing the operator $\Delta$ has the form (1), where

$$
\begin{aligned}
v & =v_{1}-v_{2} \in \mathbf{L}_{2}[0, \infty) \\
w & =\Delta\left(v_{1}\right)-\Delta\left(v_{2}\right) \in \mathbf{L}_{2}[0, \infty) .
\end{aligned}
$$

\section{ACKNOWLEDGMENT}

The authors are grateful to many people, in particular to $\mathrm{K}$. J. Åström, J. C. Doyle, U. Jönsson, and V. A. Yakubovich for comments and suggestions about this work.

\section{REFERENCES}

[1] V. M. Popov, "Absolute stability of nonlinear systems of automatic control," Automation Remote Contr., vol. 22, pp. 857-875, Mar. 1962; Russian original in Aug. 1961.

[2] V. A. Yakubovich, "Frequency conditions for the absolute stability of control systems with several nonlinear or linear nonstationary units," Automat. Telemech., pp. 5-30, 1967.

[3] G. Zames, "On the input-output stability of nonlinear time-varying feedback systems-Part I: Conditions derived using concepts of loop gain, and Part II: Conditions involving circles in the frequency plane and sector nonlinearities," IEEE Trans. Automat. Contr., vol. 11, pp. 228-238, Apr. 1966.

[4] J. C. Willems, "Dissipative dynamical systems-Part I: General theory; Part II: Linear systems with quadratic supply rates," Arch. Rational Mechanics Anal., vol. 45, no. 5, pp. 321-393, 1972.

[5] K. S. Narendra and J. H. Taylor, Frequency Domain Criteria for Absolute Stability. New York: Academic, 1973.

[6] C. A. Desoer and M. Vidyasagar, Feedback Systems: Input-Output Properties. New York: Academic, 1975.

[7] M. G. Safonov, Stability and Robustness of Multivariable Feedback Systems. Cambridge, MA: MIT Press, 1980.

[8] J. C. Doyle, "Analysis of feedback systems with structured uncertainties," Proc. Inst. Elec. Eng., vol. D-129, pp. 242-251, 1982.

[9] Yu. Nesterov and A. Nemirovski, Interior Point Polynomial Methods in Convex Programming, vol. 13 of Studies Appl. Math. Philadelphia, PA: SIAM, 1993.

[10] S. Boyd, L. El Ghaoui, E. Feron, and V. Balakrishnan, Linear Matrix Inequalities in System and Control Theory, vol. 15 of Studies Appl. Math. Philadelphia, PA: SIAM, 1994.

[11] G. Leitmann, "Guaranteed asymptotic stability for some linear systems with bounded uncertainties," J. Dynamic Syst., Measurement, Contr., vol. 101, no. 3, 1979.

[12] M. Corless and G. Leitmann, "Continuous state-feedback guaranteeing uniform ultimate boundedness for uncertain dynamic systems," IEEE Trans. Automat. Contr., vol. 26, no. 5, 1981.

[13] M. G. Safonov and M. Athans, "A multi-loop generalization of the circle criterion for stability margin analysis," IEEE Trans. Automat. Contr., vol. 26, pp. 415-422, 1981.

[14] G. Zames, "Feedback and optimal sensitivity: Model reference transformations, multiplicative seminorms and approximate inverses," IEEE Trans. Automat. Contr., vol. 26, pp. 301-320, 1981. 
[15] A. Tannenbaum, "Modified Nevanlinna-Pick interpolation of linear plants with uncertainty in the gain factor," Int. J. Contr., vol. 36, pp. $331-336,1982$

[16] A. Megretski, "Power distribution approach in robust control," in Proc. IFAC Congr., 1993.

[17] J. Shamma, "Robustness analysis for time-varying systems," in Proc. 31st IEEE Conf. Decision Contr., 1992.

[18] A. Megretski and S. Treil, "Power distribution inequalities in optimization and robustness of uncertain systems," J. Math. Syst., Estimation Contr., vol. 3, no. 3, pp. 301-319, 1993.

[19] J. C. Willems, The Analysis of Feedback Systems. Cambridge, MA: MIT Press, 1971

[20] V. A. Yakubovich, "A frequency theorem for the case in which the state and control spaces are Hilbert spaces with an application to some problems of synthesis of optimal controls-Parts I-II," Sibirskii Mat. Zh., vol. 15, no. 3, pp. 639-668, 1974; English translation in Siberian Math. $J$.

[21] A. Rantzer, "On the Kalman-Yakubovich-Popov lemma," Syst. Contr. Lett., vol. 28, no. 1, 1996

[22] G. Zames and P. L. Falb, "Stability conditions for systems with monotone and slope-restricted nonlinearities," SIAM J. Contr., vol. 6, no. 1, pp. 89-108, 1968

[23] M. K. H. Fan, A. L. Tits, and J. C. Doyle, "Robustness in presence of mixed parametric uncertainty and unmodeled dynamics," IEEE Trans. Automat. Contr., vol. 36, pp. 25-38, Jan. 1991.

[24] P. M. Young, "Robustness with parametric and dynamic uncertainty," Tech. Rep., Ph.D. dissertation, CA Tech. Inst., 1993.

[25] M. Freedman and G. Zames, "Logarithmic variation criteria for the stability of systems with time-varying gains," SIAM J. Contr., vol. 6 , pp. 487-507, 1968.

[26] A. Megretski, "Frequency domain criteria of robust stability for slowly time-varying systems," IEEE Trans. Automat. Contr., submitted.

[27] U. Jönsson and A. Rantzer, "Systems with uncertain parameters-Time variations with bounded derivatives," in Proc. Conf. Decision Contr., 1994; also Int. J. Robust Nonlinear Contr., to appear.

[28] A. Rantzer, "Uncertainties with bounded rates of variation," in Proc. Amer. Contr. Conf., 1993, pp. 29-30.

[29] __ "Uncertain real parameters with bounded rate of variation," in Adaptive Control, Filtering and Signal Processing, K. J. Åström, G. C. Goodwin, and P. R. Kumar, Eds. New York: Springer-Verlag, 1994.

[30] F. Paganini, "Set descriptions of white noise and worst case induced norms," in Proc. IEEE Conf. Decision Contr., 1993, pp. 3658-3663.

[31] B. D. O. Anderson, "A system theory criterion for positive real matrices,” SIAM J. Contr., vol. 5, pp. 171-182, 1967.

[32] R. W. Brockett and J. L. Willems, "Frequency domain stability criteria," IEEE Trans. Automat. Contr., vol. AC-10, pp. 255-261, 401-413, 1965.

[33] R. W. Brockett and H. B. Lee, "Frequency domain instability criteria for time-varying and nonlinear systems," Proc. IEEE, vol. 55, pp. 604-619, 1965.

[34] R. W. Brockett, "The status of stability theory for deterministic systems," IEEE Trans. Automat. Contr., vol. AC-11, pp. 596-606, 1966.

[35] E. I. Jury and B. W. Lee, "The absolute stability of systems with many nonlinearities," Automat. Remote Contr., vol. 26, pp. 943-961, 1965.

[36] M. G. Safonov, Stability and Robustness of Multivariable Feedback Systems. Cambridge, MA: MIT Press, 1980.

[37] M. G. Safonov and G. Wyetzner, "Computer-aided stability criterion renders Popov criterion obsolete," IEEE Trans. Automat. Contr., vol. AC-32, pp. 1128-1131, Dec. 1987.

[38] Ya. Z. Tsypkin, "Absolute stability of a class of nonlinear automatic sampled data systems," Automat. Remote Contr., vol. 25, pp. 918-923, 1964.

[39] M. Vidyasagar, Nonlinear Systems Analysis, 2nd ed. Englewood Cliffs, NJ: Prentice-Hall, 1992.

[40] A. Megretski and A. Rantzer, "System analysis via integral quadratic constraints: Part I," Department Automat. Contr., Lund Inst. Technol., Tech. Rep. TFRT-7531, 1995.

[41] J. C. Willems and R. W. Brockett, "Some new rearrangement inequalities having application in stability analysis," IEEE Trans. Automat. Contr., vol. AC-13, pp. 539-549, 1968.

[42] V. A. Kamenetskii, "Absolute stability and absolute instability of control systems with several nonlinear nonstationary elements," Automat. Remote Contr., vol. 44, no. 12, pp. 1543-1552, 1983.

[43] E. S. Pyatnitskii and V. I. Skorodinskii, "Numerical methods of Lyapunov function construction and their application to the absolute stability problem," Syst. Contr. Lett., vol. 2, no. 2, pp. 130-135, Aug. 1982.
[44] I. W. Sandberg, "A frequency-domain condition for the stability of feedback systems containing a single time-varying nonlinear element," Bell Syst. Tech. J., vol. 43, no. 3, pp. 1601-1608, July 1964.

[45] J. C. Willems, "Least squares stationary optimal control and the algebraic Riccati equation," IEEE Trans. Automat. Contr., vol. AC-16, no. 6, Dec. 1971

[46] A. Packard and J. Doyle, "The complex structured singular value," Automatica, vol. 29, no. 1, pp. 71-109, 1993.

[47] M. A. Aizerman and F. R. Gantmacher, Absolute Stability of Regulator Systems. San Francisco, CA: Holden-Day, 1964

[48] A. I. Lur'e, Some Nonlinear Problems in the Theory of Automatic Control. London: H. M. Stationery Off., 1957 (in Russian, 1951).

[49] A. Megretski, " $\mathcal{S}$-procedure in optimal nonstochastic filtering," Dept. Math., Royal Inst. Technol., S-100 44 Stockholm, Sweden, Tech. Rep. TRITA/MAT-92-0015, Mar. 1992.

[50] A. M. Lyapunov, Problème général de la Stabilité du Mouvement, vol. 17 of Ann. Math. Studies. Princeton, NJ: Princeton Univ. Press, 1947.

[51] R. Brockett, "On improving the circle criterion," in Proc. IEEE Conf. Decision Contr., 1977, pp. 255-257.

[52] V. A. Yakubovich, "Absolute stability of nonlinear control systems in critical cases-Parts I-III," Avtomaika i Telemechanika, vol. 24, no. 3, pp. 293-302, vol. 24 , no. 6 , pp. $717-731$, 1963, vol. 25 , no. 25 , pp. 601-612, 1964 (English translation in Automat. Remote Contr.).

[53] _ "The method of matrix inequalities in the theory of stability of nonlinear control systems-Parts I-III," Avtomatika i Telemechanika, vol. 25 , no. 7 , pp. 1017-1029, 1964, vol. 26, no. 4, pp. 577-599, 1964, vol. 26, no. 5, pp. 753-763, 1965 (English translation in Automat. Remote Contr.)

[54] _ , "S-procedure in nonlinear control theory," Vestnik Leningrad Univ., pp. 62-77, 1971 (English translation in Vestnik Leningrad Univ. Math., vol. 4, pp. 73-93, 1977).

[55] _ "On an abstract theory of absolute stability of nonlinear systems," Vestnik Leningrad Univ. Math., vol. 10, pp. 341-361, 1982 (Russian original published in 1977).

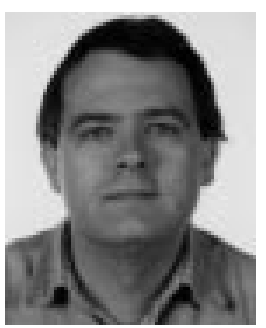

Alexandre Megretski (M'93) was born in 1963. He received the Ph.D. degree from Leningrad University in 1988 .

He held research positions at Leningrad University, Mittag-Leffler Institute, Sweden, the Royal Institute of Technology (KTH), Sweden, and the University of Newcastle, Australia. From 1993 to 1996, he was an Assistant Professor at Iowa State University. Since 1996, he has been an Assistant Professor of Electrical Engineering at Massachusetts Institute of Technology, Cambridge. His main research interests include rigorous analysis of nonlinear and uncertain systems and control design for nonminimum-phase and underactuated nonlinear systems, but also complex analysis and operator theory.

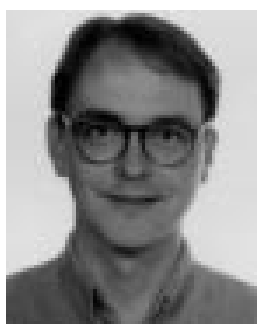

Anders Rantzer (S'92-M'94) was born in 1963 He received the M.S. degree in engineering physics and a Licentiate degree in mathematics, both from Lund Institute of Technology, Lund, Sweden, and the Ph.D. degree in optimization and systems theory from the Royal Institute of Technology (KTH), Stockholm, Sweden.

After Post-Doctoral positions at KTH and IMA, University of Minnesota, he joined the Department of Automatic Control, Lund, in 1993. His research interests include modeling, analysis, and design of control systems, particularly the effects of uncertainty and nonlinearities.

Dr. Rantzer serves as an Associate Editor of IEEE TRANSACTIONS ON Automatic Control, European Journal of Control, and Systems and Control Letters. He is a winner of the 1990 SIAM Student Paper Competition and the 1996 IFAC Congress Young Author Prize. 